\title{
A Survey of Packet Collision Minimization Algorithms in Wireless Mesh Networks
}

\author{
Tshimangadzo M. Tshilongamulenzhe, Topside E. Mathonsi and Prof Maredi I. Mphahlele \\ Department of Information Technology \\ Tshwane University of Technology
}

\begin{abstract}
This article analyzes the existing algorithms used to minimize packet collision in Wireless Mesh Networks (WMNs). We illustrate a clear sympathetic of this vigorous research area, by identifying clear guidelines used when packet collision minimization algorithms are designed. We compare strength and weakness of existing solutions and highlight the key research areas for further development. Finally, the paper presents some future perspectives on how the identified shortcomings on exiting solutions can be solved. This paper serves as a guide and a starting point for anyone willing to delve into research on packet collision minimization algorithms in WMNs.
\end{abstract}

Index Terms -Wireless Mesh Networks, Packets collision, Algorithms, Shortcomings.

\section{Introduction}

Wireless Mesh Networks have been identified as a growing area of technology for the upcoming wireless networking. WMNs is the communication technology that consists of nodes which are organized in Mesh Topology network, which provides good performance, reliability and flexibility over a wireless network. Wireless Mesh Networks can be combined with other networks such as internet, cellular, IEEE 802.11 etc., using a gateway and bridging functions in mesh network routers [1]. In WMN, the network users freely move from one point to another at any time while they are accessing the network or exchanging packets within the network. The WMNs is a network which is self-organized and repeatedly recognized by the nodes and mesh connectivity. Quality-of-service (QoS) support can be achieved by some distinguished ways in WMN [2]. WMNs uses bandwidth for communication and sending data packets from one node to another node in a form of multiple wireless hops. However, the packet collision in WMNs may occur when the packets are being transmitted at the same time using the same transmission channel, and when there is more load of network users connected or accessing the same access point using the same bandwidth. Congestion occurs every time when the routing protocol chooses the same shortest path to transmite network traffic, however, due to high traffic load on that shortest path packet collission occures. As a result, packet loss occures hence degrading the performance of WMNs [1], [3].

The full research paper is structured inn the following way: In Section II, we present packet collision overview in WMNs. In Section III, we present characteristics of WMNs. Related work is presented in Section IV; we conclude the paper.

\section{Overview of Packet Collision in WMNs}

Packet collision occurs when many network users accessing the data packets at the same time or using a high volume of data packets which exceed the capacity of the bandwidth located to the users in a wireless network. Packet loss also happens in wireless networks because of some factors wich include interference, link failure, etc. The link failure mostly happens in mobile ad hoc networks, because all nodes within a network are mobile. Collison occurs when there is a high density of data packets in a typical communication channel [4]. 


\section{Characteristics of WMNs}

As mentioned in Section I, that WMNs is getting more attractive to network users in recent years. This is because more mobile devices are becoming more affordable in a way that most network users can manage to have more than one mobile device such as Tablet, Cell Phone, Laptop, etc, and get connected to a wireless network at the same time. Mesh network topology it can also be wired but it is also more cost effective and simple to measure a mesh network using wireless access devices [5]. However, WMNs becomes the solution to many wireless network users for providing better community broadband internet access services. In WMNs, there are different types of topologies which include the infrastructure, hybrid and client, which normally made up of different devices which includes Mesh router (MRs), Mesh Clients (MCs), Acess Points (APs), Mesh Gateway Node (MGNs), etc.. The below Figure 1 shows WMNs System Architecture.

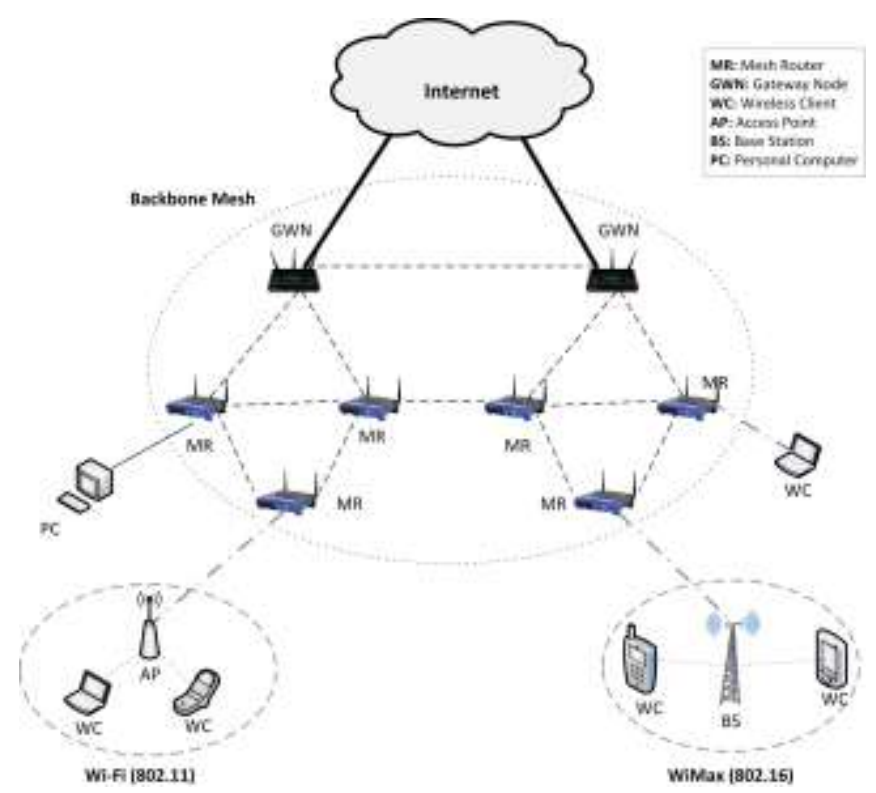

Fig. 1 Wireless Mesh Networks Systems Architecture.

WMNs is typically consist of different parts of devices such as like mesh client, mesh routers and gateways [6]. However, WMNs is in a form of mesh topology where not only the nodes or devices are to each other in a wireless manner and also use the server for data communication.

\section{Related Work}

Over the past years, the lot of work has been done based on packets collision, using different algorithms in order to minimize the packets collision in wireless mesh networks.

Jamshaid et al. [7] their aim was to provide buffer large enough to saturate the available capacity of the bottleneck collision domain that limits the capacity of the network, they proposed to use the sizing link buffers collectively that focus on the nodes within the mutual range interference of the collision domain where it will allow individual nodes to pass without any significant loss in network utilisation. The problem that they focus on was buffer sizing for TCP flows in 802.11 based in WMNs. They implemented and tested their solution using the Network simulator-2, therefore their results shows that they can reduce the end-to-end delays by $6 \mathrm{x}$ to $10 \mathrm{x}$ of the network capacity. Howerver, this increases end-to-end delay as they are buffering the size of TCP flows in 802.11.

Rangwala et al. [8] designed AIMD-based rate control algorithm to achieve the fair and efficient congestion control within wireless mesh network [8]. Rangwala et al. [8] first design the AIMD-based rate control called Wireless Control Protocol (WCP) which look at the wireless congestions within the neighborhood phenomenon and secondly they design distributed rate controller that estimates the available capacity within the neighborhood and divides the capacity contending flows, as they called it Wireless less Control Protocol with Capacity Estimation (WCPCap). The simulation results that their solution minimized the congestion within a wireless mesh network. However, solution only checks available capacity within the neighborhood, but it does not check the capacity of the current link which a node is using.

Grover et al. [1] address the problem of packets loss due to congestion, where the routing protocol always use the best shortest path for the transmission of data. Grover et al. [1] proposed to use to use the congestion control scheme based on AODV routing protocol algorithm to improve the transmission of packets within the wireless mesh network. 
Draves et al. [9] proposed to use metric for routing in Multi-radio, multi-hop wireless networks with the stationary nodes such as community wireless networks. Because there are some individual links that are available on the wireless networks, therefore, individual links are combined into a path metric called Weighted Cumulative ETT (WCETT) that openly books for the interference among links that use the same transmission channels. The main goal of this metric is to choose the best high-throughput path between the sources and the destination. The simulation result showed better network performance when their solution is applied, however, their does not check the link load of the path with high-troughput and this increases end-to-end delay in lager WMNs.

Waharte et al. [10] proposed the problem of traffic flows allocation in a wireless network, and they solution was to implement the interference-aware liner-programming formulation of the Min and Max congestion with and without constraints on the path length. They use network simulator-2 (NS-2) to simulate their results and the simulations results showed that their solution can select the best path with least link interfarence. However, their solution is only interference-aware while link load metric is also very important when it comes to packet collision miminization in WMNs.

Rao et al. [11] proposed the metric performance evaluation of routing protocols where there is packets loss and congestion based on the transmission channel. Their proposed solution was to use the ad hoc on demand distance vector (AODV) and Destination-Sequenced and Distance Vector (DSDV) routing protocol to minimize their problem. Their solution was tested using network simulator 2 (NS-2) and the simulation results showed better network performance. However, their solution does not check the link load and link interfarence before selecting the best path in order to aviod parket collision in WMNs.

Zakaria et al. [12] proposed the minimization of end-to-end delay within the wireless mesh network, and their solution was to use the hybrid wireless mesh protocol (HWMP) to minimize the delay of the end-to-end point. They use network simulator-2 (NS-2) to test their solution. The simulation results showed that their solution can minimize end-to-end delay, however, their solution does not check link interfarence which increases packet collision in WMNs.

Nassereddine et al. [13] they proposed to adventure the trade-off between network cost and the overall network performance of the wireless mesh network, and their solution was to introduce the new multi-objective model for all nodes placement optimization in Multi-Radio Multi-Channel WMNs. They use OPNET to test their solution. The simulation results showed that their solution can better place nodes in a Multi-Radio Multi-Channel WMNs, however, nodes placed anywhere in Multi-Radio Multi-Channel WMNs before checking the link load and link interfarence which increases packet collision during packet transmission.

Fonseca [14] proposed to minimize the interference between the transmissions of neighboring nodes, their solution was to use novel clustering algorithms and complementary protocols. NS-2 was used to test their solution. The silumation results show that their solution reduced interfarence between the transmissions of neighboring nodes, however, their solution did not solve the issue of packet collision as end-to-end delay remained as a problem with their solution.

Mathonsi \& Kogeda [15], [16] proposed Dijkstra-Transferrable Payoff (DTP) algorithm in order to solve high network latency, and link congestion network problems which led to packet collision and high number of lost packets during packet transmission. The enhanced bandwidth sharing scheme was designed by integrating transferrable payoff coalitional game theory and the standard-Dijkstra algorithm. DTP algorithm introduced transferrable payoff coalitional game theory into hop by hop routing and channel bandwidth allocation. The integration on DTP algorithm did not add computational complexity, in the sense that, rather than splitting the processes and experiences additional computational duty cycles, it merged the processes into one and execute the resulting functionality using one duty cycle. NS-2 was used to simulator their solution and the simulation results showed that high network latency and link congestion were minimized, as a result, packet collision and high number of lost packets during packet transmission were also minimized [15], [16]. However, the time taken to select best paths in a network and for bandwidth allocation was increased through merging of the two processes. This increased the end-to-end delay and a result packet collision still occurred.

\section{Conclusion and Future Work}

In this paper, we presented an insight of WMNs, by stating how WMNs works and its characteristics. Thereafter, we highlighted a number of related works on WMNs that have the aim of reducing packet collision and presented their shortcomings. We hope that this inclusive survey act as a starting point for new research in packet collision minimization algorithms in WMNs. In future, we will use a simulation to test the under design enhanced algorithm and show an improved network throughput and better QoS as compared to existing algorithms. The under designed algorithm will use $802.11 \mathrm{ac}$ and multi-hop transmission packet collision to minimize packet collision by arranging the management frames sent on an IEEE 802.11 wireless link. 


\section{Bibliography}

[1] P. Grover, P. Singh, and S. Rani, "Congestion Reduction in Wireless Mesh Networks," International Journal of Computer Applications, vol. 124, 2015.

[2] C.Y. Liu, B. Fu, and H.-J. Huang, "Delay minimization and priority scheduling in wireless mesh networks," Wireless Networks, vol. 20, pp. 1955-1965, 2014. https://doi.org/10.1007/s11276-014-0721-2

[3] A. Adekiigbe, K. Abu-Bakar, and O. Ogunnusi, "A review of cluster-based congestion control protocols in wireless mesh networks," Int. J. Comput. Sci, vol. 8, pp. 42-50, 2011.

[4] M. N. Sudha, M. L. Valarmathi, G. Rajsekar, M. K. Mathew, N. Dineshraj, and S. Rajbarath, "Minimization of collision in an energy constrained wireless sensor network," Wireless Sensor Network, vol. 1, p. 350, 2009. https://doi.org/10.4236/wsn.2009.14043

[5] L. Ndlovu, M. Lall, and O. P. Korea, "A review of service discovery schemes in wireless mesh networks," in 2016 IST-Africa Week Conference, 2016, pp. 1-7

[6] S. Kumar, G. R. Murthy, and N. Chilamkurti, "Cooperative Mesh Networks," in Next-Generation Wireless Technologies, ed: Springer, 2013, pp. 7-13. https://doi.org/10.1007/978-1-4471-5164-7_2

[7] K. Jamshaid, B. Shihada, L. Xia, and P. Levis, "Buffer sizing in 802.11 wireless mesh networks," in 2011 IEEE Eighth International Conference on Mobile Ad-Hoc and Sensor Systems, 2011, pp. 272-281. https://doi.org/10.1109/MASS.2011.34

[8] S. Rangwala, A. Jindal, K.-Y. Jang, K. Psounis, and R. Govindan, "Understanding congestion control in multi-hop wireless mesh networks," in Proceedings of the 14th ACM international conference on Mobile computing and networking, 2008, pp. 291-302. https://doi.org/10.1145/1409944.1409978

[9] R. Draves, J. Padhye, and B. Zill, "Routing in multi-radio, multi-hop wireless mesh networks," in Proceedings of the 10th annual international conference on Mobile computing and networking, 2004, pp. 114-128. https://doi.org/10.1145/1023720.1023732

[10] S. Waharte, A. Farzan, and R. Boutaba, "Min-max congestion in interference-prone wireless mesh networks," in 2007 IEEE International Conference on Communications, 2007, pp. 3746-3751. https://doi.org/10.1109/ICC.2007.617

[11] S. S. N. Rao, Y. S. Krishna, and K. N. Rao, "Performance Evaluation of routing protocols in Wireless Mesh networks," International Journal of Computer Applications, vol. 68, 2013.

[12] A. Zakaria, H. Mohamad, N. Ramli, and M. Ismail, "Performance evaluation of routing protocols in wireless mesh network," in Advanced Communication Technology (ICACT), 2013 15th International Conference on, 2013, pp. 1111-1115.

[13] T. M. B. Nassereddine, A. Haqiq, and S. Bennani, "Multi-Objective Particle Swarm Optimization for Facility Location Problem in Wireless Mesh Networks," arXiv preprint arXiv:1308.5807, 2013.

[14] C. N. Fonseca, "Multipath Routing in Wireless Mesh Networks," Ph. D. Dissertation, 2010.

[15]T. E. Mathonsi and O. P. Kogeda, "Implementing Wireless Network Performance Optimization for Small and Medium Enterprises, " Pan African Conference on Science, Computing and Telecommunications (PACT2014) proceedings, 2014. https://doi.org/10.1109/scat.2014.7055139

[16] T. E. Mathonsi and O. P. Kogeda, "Enhanced Bandwidth Sharing Scheme for Small and Medium Enterprises," International Association of Engineers (IAENG) World Congress on Engineering and Computer Science 2014 (WCECS 2014) proceedings, pp. $765-770,2014$. 